\title{
Hemodynamic characteristics of the right ventricle following gradient pulmonary artery banding in rats
}

\author{
JIYANG SONG ${ }^{1}$, SHUTONG SHEN $^{2}$, YING WEI $^{1}$, WENLI LI $^{1}$ and NAN WANG ${ }^{1}$ \\ ${ }^{1}$ Department of Cardiology, Gansu Provincial Hospital, Lanzhou, Gansu 730000; \\ ${ }^{2}$ Department of Cardiology, Zhongshan Hospital, Fudan University, Shanghai 200030, P.R. China
}

Received April 5, 2011; Accepted Sept 3, 2021

DOI: 10.3892/wasj.2021.125

\begin{abstract}
Right ventricle/ventricular (RV) failure induced by sustained pressure overload is a major contributor to morbidity and mortality in several cardiopulmonary disorders. Reliable and reproducible animal models of RV failure are crucial for investigating disease mechanisms and the effects of potential therapeutic strategies. In the present study, in order to establish a rat model of acute RV failure, the hemodynamic characteristics of the RV and the carotid artery in rats with varying degrees of pulmonary artery banding (PAB) were analyzed. For this purpose, rats were divided into 6 groups as follows: The control (0\%), PAB (1-30\%), PAB (31-60\%), PAB $(61-70 \%)$, PAB (71-80\%), or PAB (100\%). RV pressure (RVP) was measured using right heart catheterization when the pulmonary artery was ligated. The results revealed that the RVP gradually increased with the increasing degrees of banding; however, when the occlusion level exceeded $70 \%$, a high-pressure state was only maintained for a few minutes or seconds, and the RVP then rapidly declined to below normal pressure levels, which was particularly evident in the PAB (100\%) group. On the whole, the present study demonstrates that RVP responds differently to changes in the occlusion level, and that $>70 \%$ ligation is a successful model of acute right heart failure. These results may have important implications for therapeutic strategies to prevent acute right heart failure.
\end{abstract}

Correspondence to: Dr Nan Wang, Department of Cardiology, Gansu Provincial Hospital, 204 Donggang West Road, Lanzhou, Gansu 730000, P.R. China

E-mail: wangnan2015@163.com

Abbreviations: RV, right ventricle/ventricular; LV, left ventricle/ventricular; PAB, pulmonary arterial banding; RVP, right ventricular pressure; $\mathrm{PAH}$, pulmonary arterial hypertension; $\mathrm{RVH}$, right ventricle hypertrophy; $\mathrm{MCT}$, monocrotaline

Key words: hemodynamic characteristics, right ventricular hypertrophy, right ventricular failure, pulmonary gradient, pulmonary artery banding, rats

\section{Introduction}

Heart failure is a common, costly, disabling and lethal disease (1). Heart failure substantially reduces the physical and mental health of patients, resulting in a markedly compromised quality of life $(2,3)$. In developing and developed countries $\sim 2 \%$ of adults suffer from heart failure; however, this rate increases to $6-10 \%$ among individuals aged $>65$ years (4-7). Left heart failure has been extensively investigated and a number of research hypotheses have been developed. However, right heart failure is characterized by increasing rates of morbidity and mortality, and the exact underlying pathological mechanisms remain elusive. Right ventricle/ventricular (RV) dysfunction has been shown to be the main outcome of pulmonary hypertension and congenital heart disease.

Pulmonary arterial hypertension (PAH) may affect individuals of any age, from newborns to adults. PAH leads to the dysfunction of both the pulmonary vasculature and the heart. Acute PAH results in severe RV failure. RV adaptation and ventricular remodeling occur following changes in the pulmonary vasculature. RV hypertrophy (RVH) follows PAH owing to compensatory mechanisms to the increased afterload. However, persistent overload results in RV dysfunction and failure. The ventricular pressure-volume relationship, and changes in wall thickness and geometry, are involved in RV remodeling. Recently, a variety of preclinical right heart failure models have emerged, and these acute (pulmonary trunk banding) (8-10) and chronic models [monocrotaline (MCT)-induced right heart failure] $(11,12)$ have been employed in research on right heart failure. However, the MCT model involves several key pathological mechanisms of human PAH other than RV failure, including vascular remodeling, the proliferation of smooth muscle cells, endothelial cell dysfunction and the upregulation of inflammatory cytokines (13). Accordingly, it is not possible to conclude whether an improvement in RV function following an intervention is secondary to the afterload-reducing pulmonary vascular effects, or whether it is caused by direct effects on the RV. At present, a more effective model of acute right heart failure is considered to be pulmonary artery banding (PAB), in which a model is created using a prolene suture tied tightly around a needle alongside the main pulmonary artery. Following the subsequent rapid removal of the needle, a fixed constricted opening is created in the lumen, equal to the diameter of the 
needle (10). However, this method is associated with major issues and concerns: First, the diameter of the banding is not exactly the same as the outer diameter of the needle, as the ligature is tied around both the needle and the pulmonary trunk (10). Second, there may be significant variations as to how tightly the knot is tied, thus rendering it difficult to exactly reproduce a certain degree of banding. Third, the diameters of the main pulmonary trunk of rats of different body weights differ significantly, as shown by a preliminary experiment (data not shown). The effect of different degrees of PAB on RV and arterial hemodynamic characteristics has not been determined for this reason; furthermore, whether this model can be used as an acute model of right heart failure remains unknown.

Therefore, the aim of the present study was to observe the hemodynamic characteristics of the RV and carotid artery under varying degrees of PAB in rats, in order to establish a rat model of progressive acute RV pressure overload.

\section{Materials and methods}

Establishment of rat model of $R V$ failure using $P A B$. A total of 32 male 8-week-old Sprague-Dawley rats (weight, 200-300 g) were purchased from the Animal Core Facility of Nanjing Medical University. The rats were housed in a temperature and humidity-controlled facility $\left(25 \pm 1^{\circ} \mathrm{C}\right.$ with a relative humidity of 40-70\%) and provided with ad libitum access to food and water. Illumination was regulated with 12 -h light/dark cycles. All experimental procedures conformed to the Guide for the Care and Use of Laboratory Animals published by the US National Institutes of Health. The study was approved by the Ethics Committee of Gansu Provincial Hospital.

A rat model of RV was established using varying degrees of PAB to measure RV and carotid artery pressure. The rats were anesthetized with an intraperitoneal injection of pentobarbital (50 mg/kg body weight) and ventilated with air using a volume-controlled respirator $(4 \mathrm{ml}, 70$ cycles $/ \mathrm{min})$. The rats were divided into 6 groups according to the degree of PAB (Fig. 1) as follows: The control $(n=5)$; PAB (1-30\%), $n=4$; PAB (31-60\%), n=6; PAB (61-70\%), $n=5 ;$ PAB (71-80\%), $n=4$; and PAB $(100 \%), n=3$.

The pulmonary arterial trunk was carefully exposed and the RV was isolated through a middle thoracotomy. The RV pressure and the rate of increase in RV pressure (dp/dt) were measured using PowerLab 8/35 (ADInstruments). The carotid artery was isolated through a midline incision in the neck, and the pressure was then measured using PowerLab 8/35. Of the 32 rats, 5 died from intraoperative bleeding; in other similar research, the mortality rate was $<1$ in 6 (9). The euthanasia (euthanasia was performed by an intraperitoneal injection of pentobarbital, $300 \mathrm{mg} / \mathrm{kg}$ body weight) of experimental animals was considered due to the following factors: The end of the experiment, for the provision of blood or other tissue organs for research needs or when the animal's pain and distress were more than expected (obvious painful struggle after the anesthesia). The procedural surgery success rate was $84.4 \%$. The pulmonary arterial trunk was clipped using a small arterial clip (Fig. 2) when the main pulmonary artery was clearly exposed and measured. The inner diameter of the clip was equal to the diameter of the compressed pulmonary artery when the clip was compressed, and could be calibrated by using, for example, a needle with a known outer diameter. The degree of PAB was represented as the stenosis rate of the cross-sectional area.

Histopathological analysis. At the end of the study, all rats were anaesthetized by an intraperitoneal injection of $50 \mathrm{mg} / \mathrm{kg}$ pentobarbital sodium, and then sacrificed by cervical dislocation. Digital recordings of the RV and carotid artery pressures were obtained using a Mikro-Tip ${ }^{\circledR}$ sensor (Millar Instruments) prior to euthanasia. The hearts were rapidly removed, and the fresh ventricular tissues were immediately blotted dry and weighed to examine the association between heart weight and pulmonary artery diameter. Tissue specimens for pathological analysis were obtained from whole hearts, the tissues were fixed in neutral formalin, embedded in paraffin, cut into $5-\mu \mathrm{m}$-thick cross sections and stained with hematoxylin $\left(25^{\circ} \mathrm{C}\right.$, 3-8 min) (Solarbio) and eosin $\left(25^{\circ} \mathrm{C}, 1-3 \mathrm{~min}\right)$ (Beijing Solarbio Science \& Technology Co., Ltd.) for morphological analysis, including the measurement of the cross-sectional area (CSA) of the RV and left ventricle (LV) using ImageJ 1.45 software (National Institutes of Health).

Statistical analysis. All data are expressed as the mean \pm SEM. Statistical analyses were performed using Statistical Program for Social Sciences 20.0 (IBM SPSS Inc.). The data were examined using the D'Agostino-Pearson normality test to confirm normal distribution, after which Levene's test was used to confirm the homogeneity of variance. The differences in the parameters between the normal control and PAB groups were analyzed using one-way ANOVA followed by Tukey's post hoc test. A P-value $<0.05$ was considered to indicate a statistically significant difference.

\section{Results}

Baseline characteristics of the rats included in the study. No marked differences were found between the body weight, heart weight and pulmonary artery diameter in the different groups of rats (Table I).

Change in the $R V$ area following gradient PAB. Gross morphological changes clearly demonstrated that the size of the RV cavity increased following PAB, as illustrated in Fig. 1. RVP follows PAH owing to compensatory mechanisms to the increased afterload. Persistent overload results in RV dysfunction and failure and, eventually, death from acute right heart failure ensues within $20 \mathrm{~min}$. Therefore, the RV area was evaluated following PAB for a period of $20 \mathrm{~min}$. The RV area increased with the increasing ligation degree (Fig. 1), particularly in the PAB (61-70\%), PAB (71-80\%) and PAB (100\%) groups, and the RV/LV CSA and RV/(RV + LV) CSA were significantly higher compared with those of the control group rats $(\mathrm{P}<0.01$; Fig. 3$)$.

Hemodynamic characteristics of the $R V$ following gradient $P A B$. As shown in Fig. 4A, the maximum pressure of the RV did not markedly differ between the PAB (1-30\%) and control groups; however, that of the PAB (31-60\%) and PAB (61-70\%) groups was significantly higher compared with that of the 
Table I. Baseline characteristics of the rats and procedural features.

\begin{tabular}{lcccccc}
\hline & \multicolumn{5}{c}{ Groups } \\
\cline { 2 - 6 } Characteristics & Control & PAB (1-30\%) & PAB (31-60\%) & PAB (61-70\%) & PAB (71-80\%) & PAB (100\%) \\
\hline No. of rats & 5 & 4 & 6 & 5 & 4 & 3 \\
Body weight $(\mathrm{g})$ & $242.7 \pm 22.27$ & $220.48 \pm 12.77$ & $234.23 \pm 23.4$ & $225.17 \pm 7.5$ & $234.7 \pm 12.76$ & $240.77 \pm 13.41$ \\
Heart weight $(\mathrm{g})$ & $0.77 \pm 0.08$ & $0.70 \pm 0.06$ & $0.74 \pm 0.06$ & $0.71 \pm 0.07$ & $0.70 \pm 0.07$ & $0.73 \pm 0.05$ \\
PA diameter $(\mathrm{mm})$ & $3.50 \pm 0.52$ & $3.26 \pm 0.33$ & $3.39 \pm 0.20$ & $3.52 \pm 0.32$ & $3.49 \pm 0.49$ & $3.18 \pm 0.29$ \\
\hline
\end{tabular}

No significant differences were observed between the groups for any of the features. PA, pulmonary artery; PAB, PA banding.

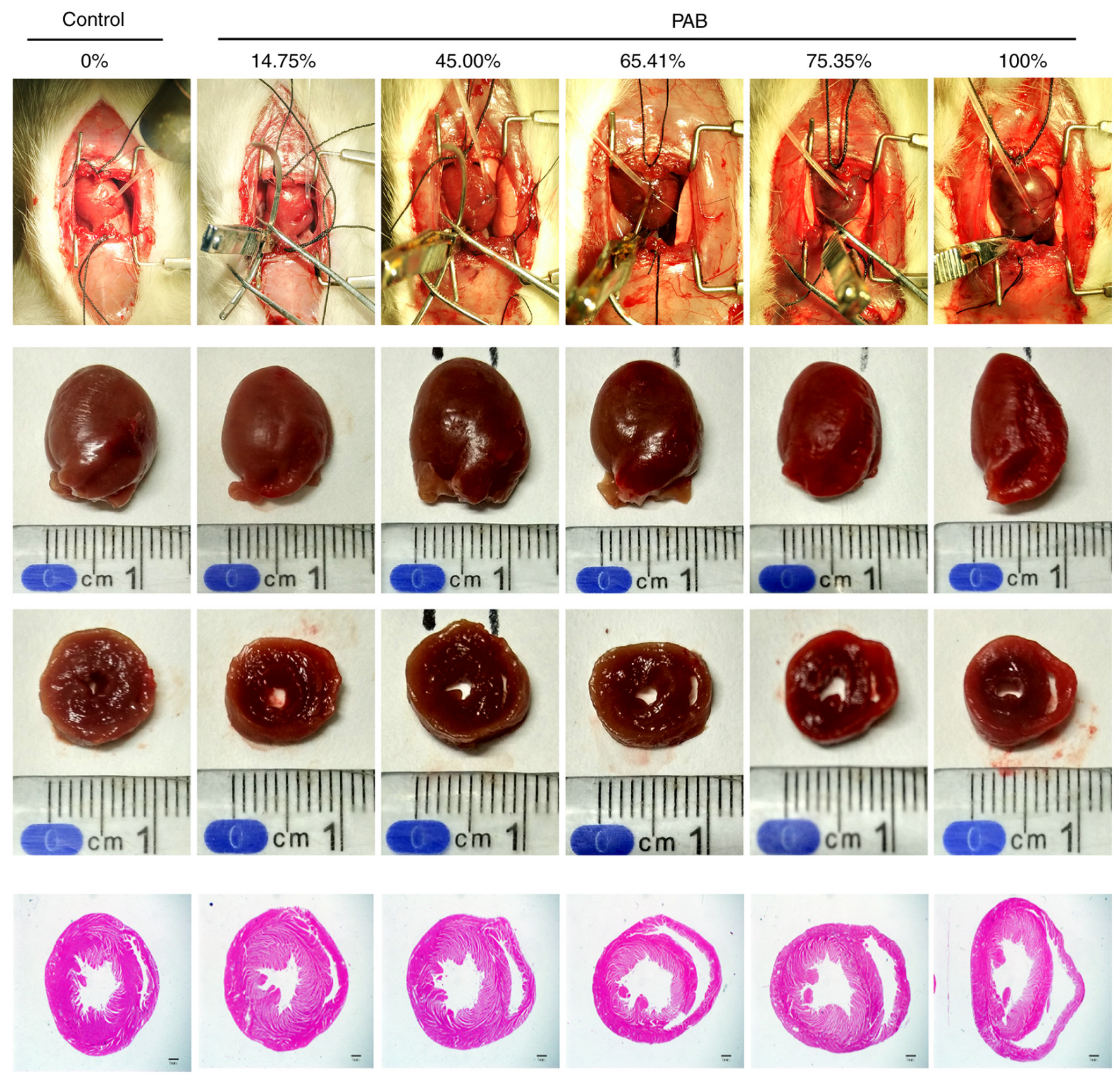

Figure 1. The specific degree of ligation of a specific rat is illustrated. For example, 65.41\% represents the degree of ligation in a rat in the 61-70\% group. The first row of images demonstrates the measurement of right ventricular pressure under pulmonary artery ligation; the second row of images demonstrates heart specimens from the rats; the third row of images demonstrates the cross section of the heart; the fourth row of images demonstrates the H\&E stained sections the cross section of the heart. The RV area increased following gradient PAB. Scale bar, $1 \mathrm{~mm}$. RV, right ventricle/ventricular; PAB, pulmonary arterial banding. 

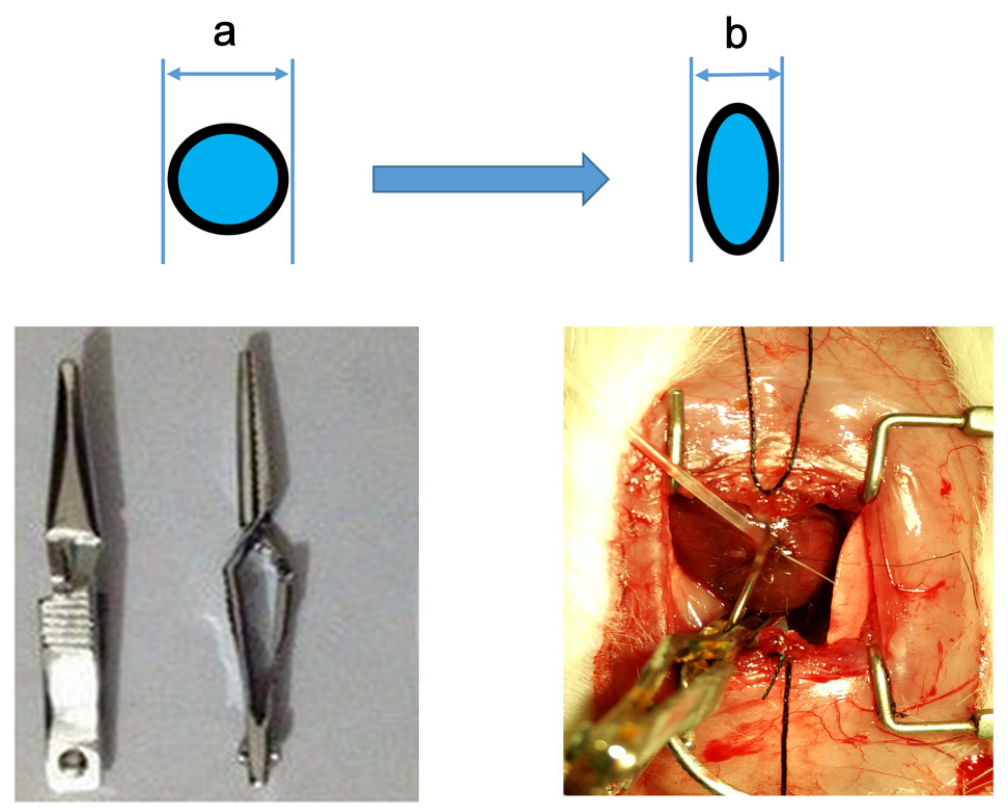

Figure 2. The degree of PAB was adjusted with the use of an adjustable arterial clamp with needles of various diameters. The degree of PAB was calculated as follows: Degree of PAB $=\left[1-\left(\pi a b-\pi b^{2}+2 b^{2}\right) /\left(2 a^{2}\right)\right] \times 100 \%$, where ' $a$ ' is the diameter of the needle and ' $b$ ' is the diameter of the pulmonary trunk. PAB, pulmonary artery banding.
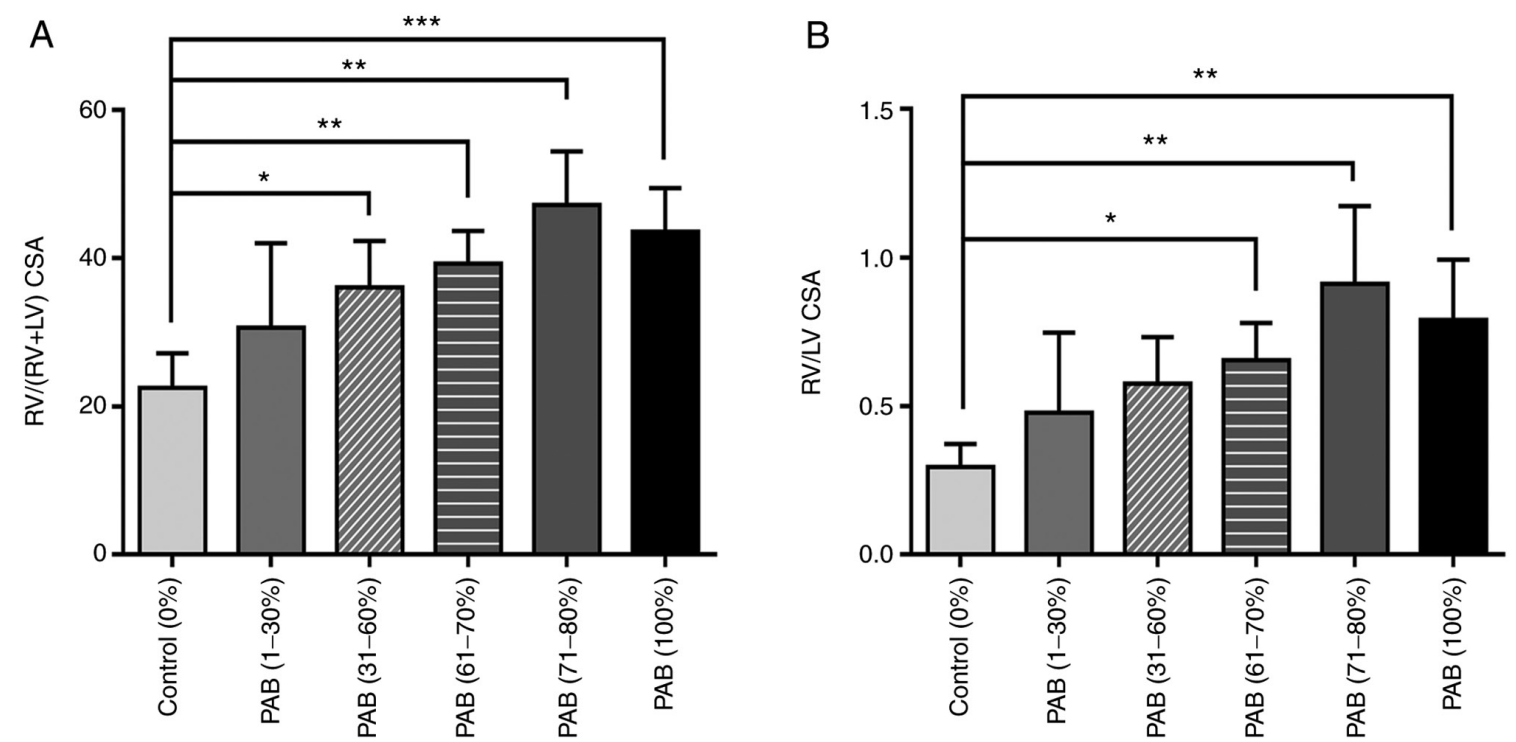

Figure 3. (A) RV/LV cross-sectional area and (B) RV/(RV+LV) cross-sectional area. Data are presented as the mean \pm SEM. One-way ANOVA followed by Tukey's post hoc test. ${ }^{*} \mathrm{P}<0.05,{ }^{* *} \mathrm{P}<0.01$ and ${ }^{* * *} \mathrm{P}<0.001$ (all PAB groups vs. control). RV, right ventricle; $\mathrm{LV}$, left ventricle; PAB, pulmonary artery banding.

control group. However, the pressure of the RV increased rapidly and decreased rapidly in $2 \mathrm{sec}$ when the degree of ligation was $71-100 \%$ [PAB (71-80\%) and PAB (100\%) groups]. As regards the minimum pressure of the RV, no marked differences were observed between the PAB (1-30\%), PAB (31-60\%), PAB (61-70\%) and control groups; however, the minimum RV pressure in the PAB (71-80\%) and PAB (100\%) groups was significantly greater compared with that in the control group $(\mathrm{P}<0.01)$ (Fig. 4B).

The carotid arterial blood pressure did not differ significantly between the PAB (1-30\%), PAB (31-60\%), PAB (61-70\%) and the control groups; however, the arterial systolic blood pressure decreased rapidly in $2 \mathrm{sec}$ when the degree of ligation was $71-100 \%$ [PAB (71-80\%) and PAB (100\%) groups] $(\mathrm{P}<0.01$; Fig. 5A). The carotid arterial diastolic blood pressure exhibited a similar trend (Fig. 5B). The mean heart rate decreased rapidly in $2 \mathrm{sec}$ when the degree of ligation was 71-100\%; however, no marked differences were observed between the PAB (1-30\%), PAB (31-60\%), PAB (61-70\%) and the control groups (Fig. 5C).

The positive rate of RV pressure increase (+dp/dt) did not markedly differ between the PAB (1-30\%), PAB (31-60\%), $\mathrm{PAB}(61-70 \%)$ and the control groups following PAB; however, the $+\mathrm{dp} / \mathrm{dt}$ increased rapidly and decreased rapidly in $2 \mathrm{sec}$ when the degree of ligation was $71-100 \%(\mathrm{P}<0.01$; Fig. 6A). Furthermore, the negative rate of increase in RV pressure (-dp/dt) 

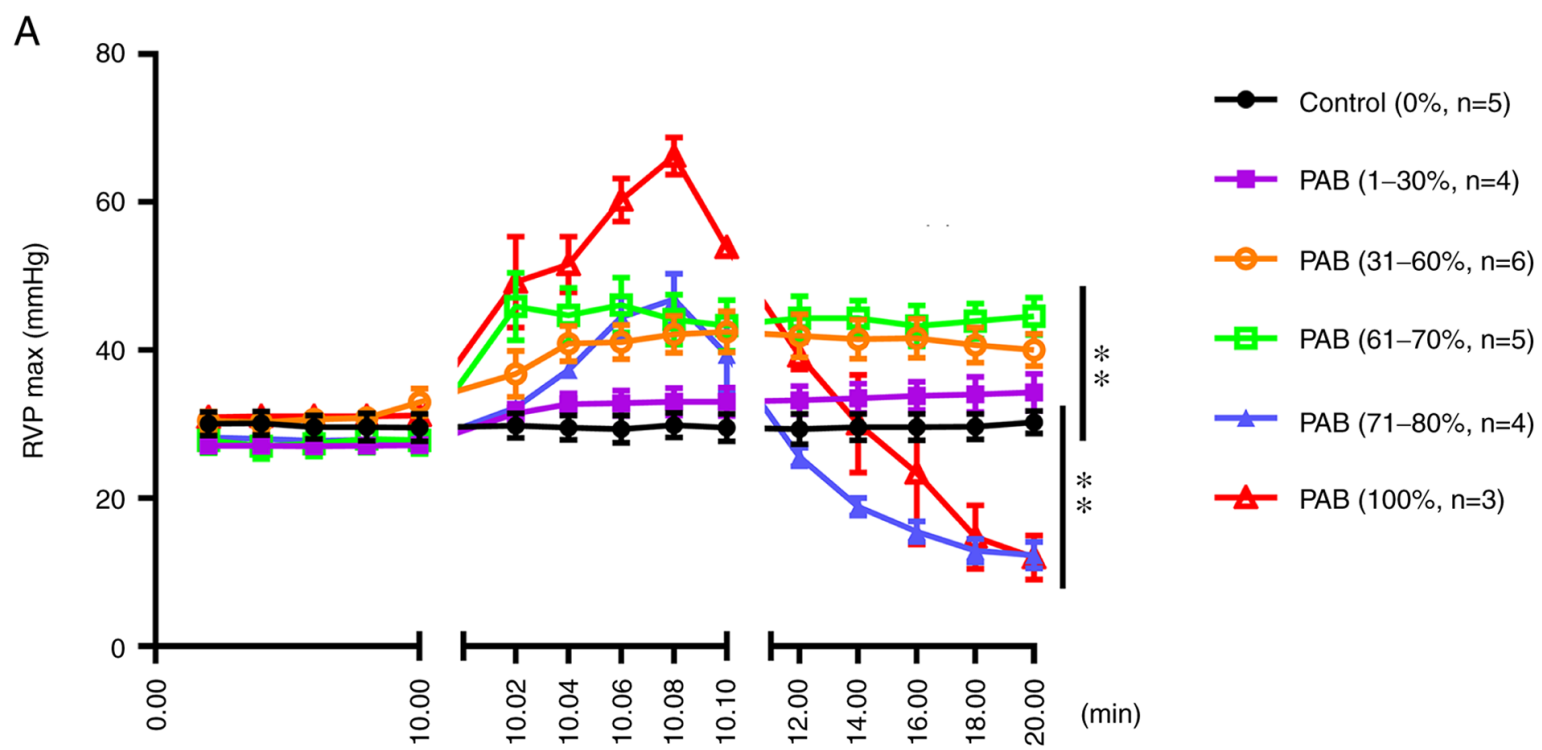

B

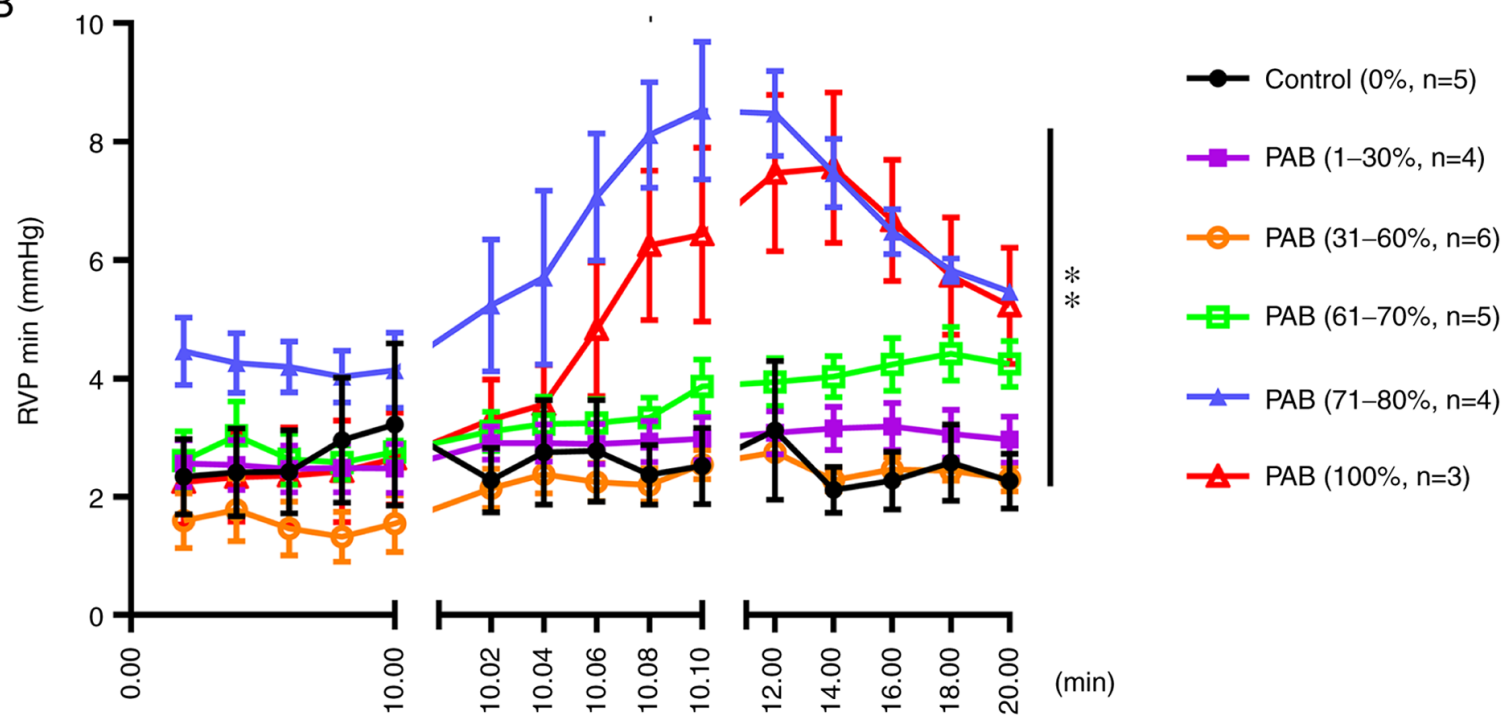

Figure 4. (A) Maximum pressure of the RV following gradient PAB. The results of statistical analysis of the differences between groups are as follows in detail: Control vs. PAB (1-30\%), not significant; control vs. PAB (31-60\%), P<0.01; control vs. PAB (61-70\%), P<0.01; control vs. PAB (71-80\%), P<0.01; control vs. $\mathrm{PAB}(100 \%), \mathrm{P}<0.01$. (B) Minimum pressure of the RV following gradient PAB. The results of statistical analysis of the differences between groups are as follows in detail: Control vs. PAB (1-30\%), not significant; control vs. PAB (31-60\%), not significant; control vs. PAB (61-70\%), not significant; control vs. PAB (71-80\%), $\mathrm{P}<0.01$; control vs. $\mathrm{PAB}(100 \%), \mathrm{P}<0.01$. RV, right ventricle/ventricular; RVP, right ventricular pressure; $\mathrm{PAB}$, pulmonary artery banding. ${ }^{* *} \mathrm{P}<0.01$.

did not markedly differ between the PAB (1-30\%), PAB (31-60\%), PAB $(61-70 \%)$ and the control groups, although the values in the PAB (71-80\%) and PAB (100\%) groups were significantly higher compared with those in the control group $(\mathrm{P}<0.01$; Fig. 6B).

\section{Discussion}

In animal models, right heart failure is difficult to induce and define, as there are neither objective cut-off values of cardiac or ventricular dysfunction nor changes in pressure, dimensions, or volume that can be reliably used for identification. In the present study, the hemodynamic characteristics of the RV and carotid artery in rats with varying degrees of PAB were observed in order to provide a basis for future research.

Based on the data obtained herein, acute right heart failure was successfully mimicked with PAB at 71-80\% (RV pressure increased and then rapidly decreased). Data from previous studies have suggested that the pulmonary hypertension model was established by PAB with a fixed degree of ligation in animals of the same week of age $(10,14)$. It was considered by the authors that the diameters of the main pulmonary arteries of rats in the same age group differed, although no significant difference was found, and the diameter of the pulmonary artery significantly affects the degree of ligation. It is not accurate to determine the degree of ligation based on the age of the rat. This approach is likely to result in non-repeatability. Therefore, the authors directly measured the diameter of the pulmonary artery and then determined the degree of ligation.

In another approach, as demonstrated in previous studies, a rat model of MCT-induced RV failure based upon a single intraperitoneal MCT injection (usually $60 \mathrm{mg} / \mathrm{kg}$ ) was applied, resulting in the development of PAH after $3-4$ weeks $(11,15,16)$. MCT-induced RV failure is similar to human PAH in terms 

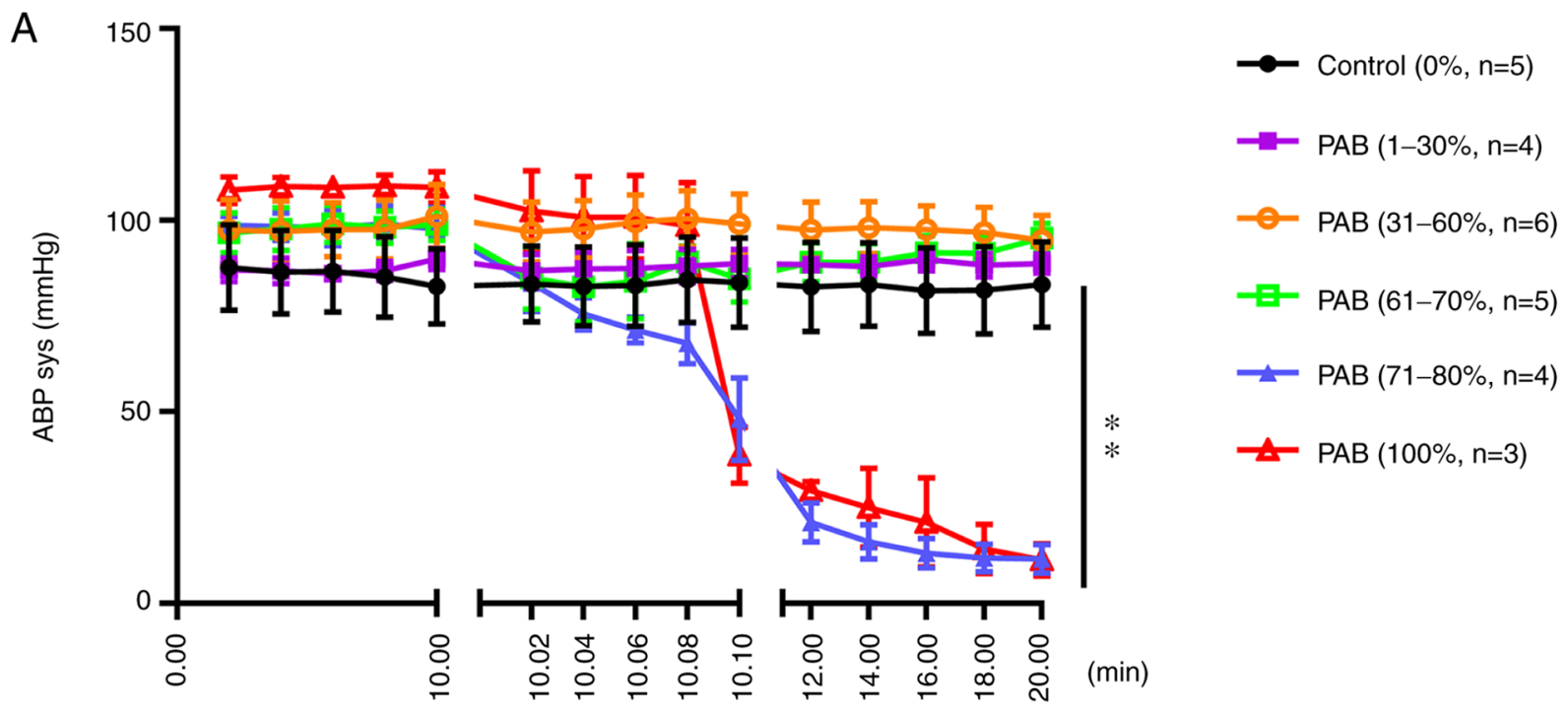

B
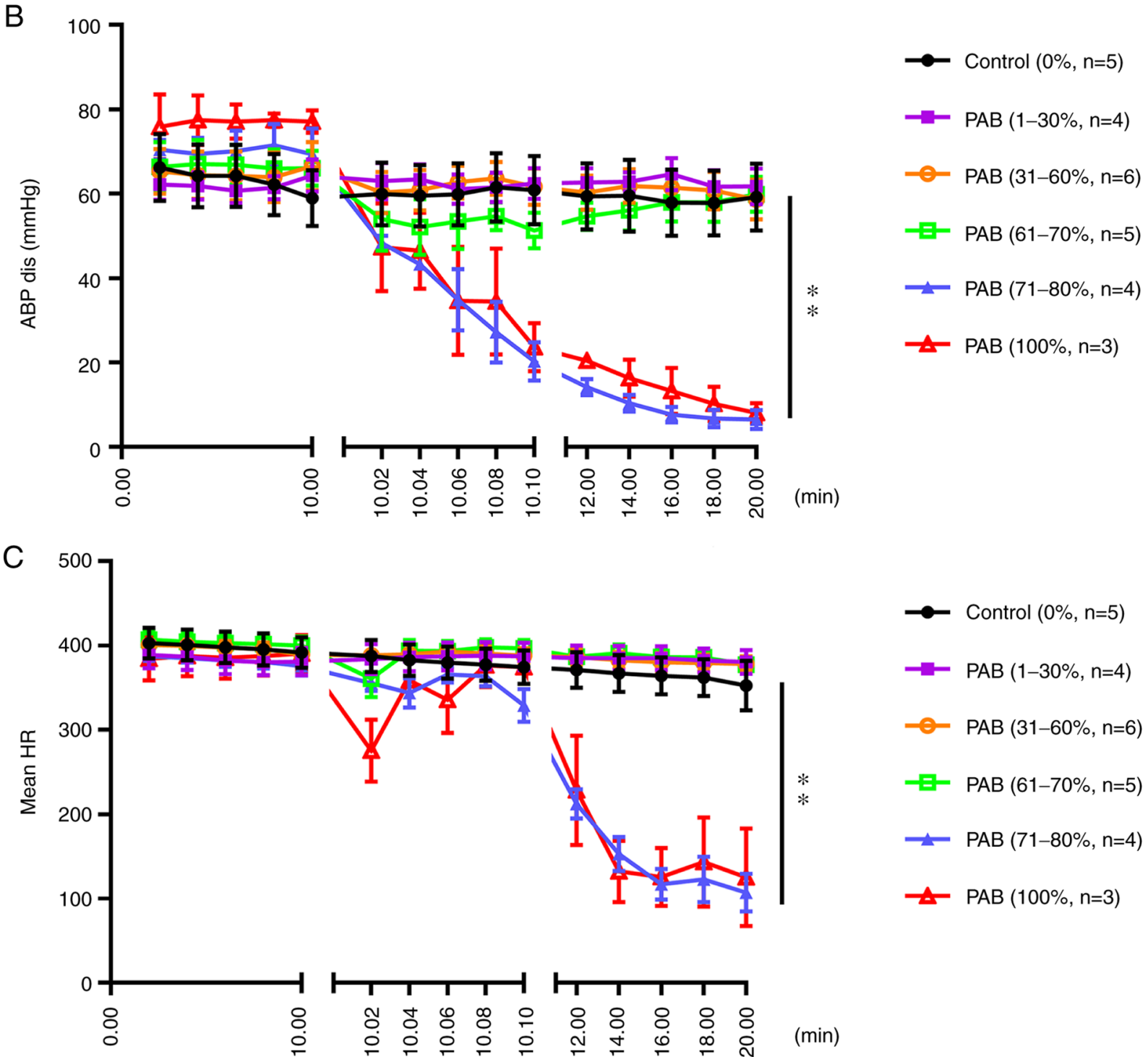

(min)

Figure 5. (A) Carotid arterial systolic blood pressure following gradient PAB. The results of statistical analysis of the differences between groups are as follows in detail: Control vs. PAB (1-30\%), not significant; control vs. PAB (31-60\%), not significant; control vs. PAB (61-70\%), not significant; control vs. PAB (71-80\%), P<0.01; control vs. PAB (100\%), P<0.01. (B) Carotid arterial diastolic blood pressure following gradient PAB. The results of statistical analysis of the differences between groups are as follows in detail: Control vs. PAB (1-30\%), not significant; control vs. PAB (31-60\%), not significant; control vs. PAB (61-70\%), not significant; control vs. PAB (71-80\%), P<0.01; control vs. PAB (100\%), P<0.01. (C) Mean heart rate following gradient PAB. The results of statistical analysis of the differences between groups are as follows in detail: Control vs. PAB (1-30\%), not significant; control vs. PAB (31-60\%), not significant; control vs. PAB (61-70\%), not significant; control vs. PAB (71-80\%), P $<0.01$; control vs. PAB (100\%), P $<0.01$. PAB, pulmonary artery banding; ABP sys, systolic arterial blood pressure; ABP dis, diastolic arterial blood pressure; HR, hear rate. ${ }^{* *} \mathrm{P}<0.01$. 


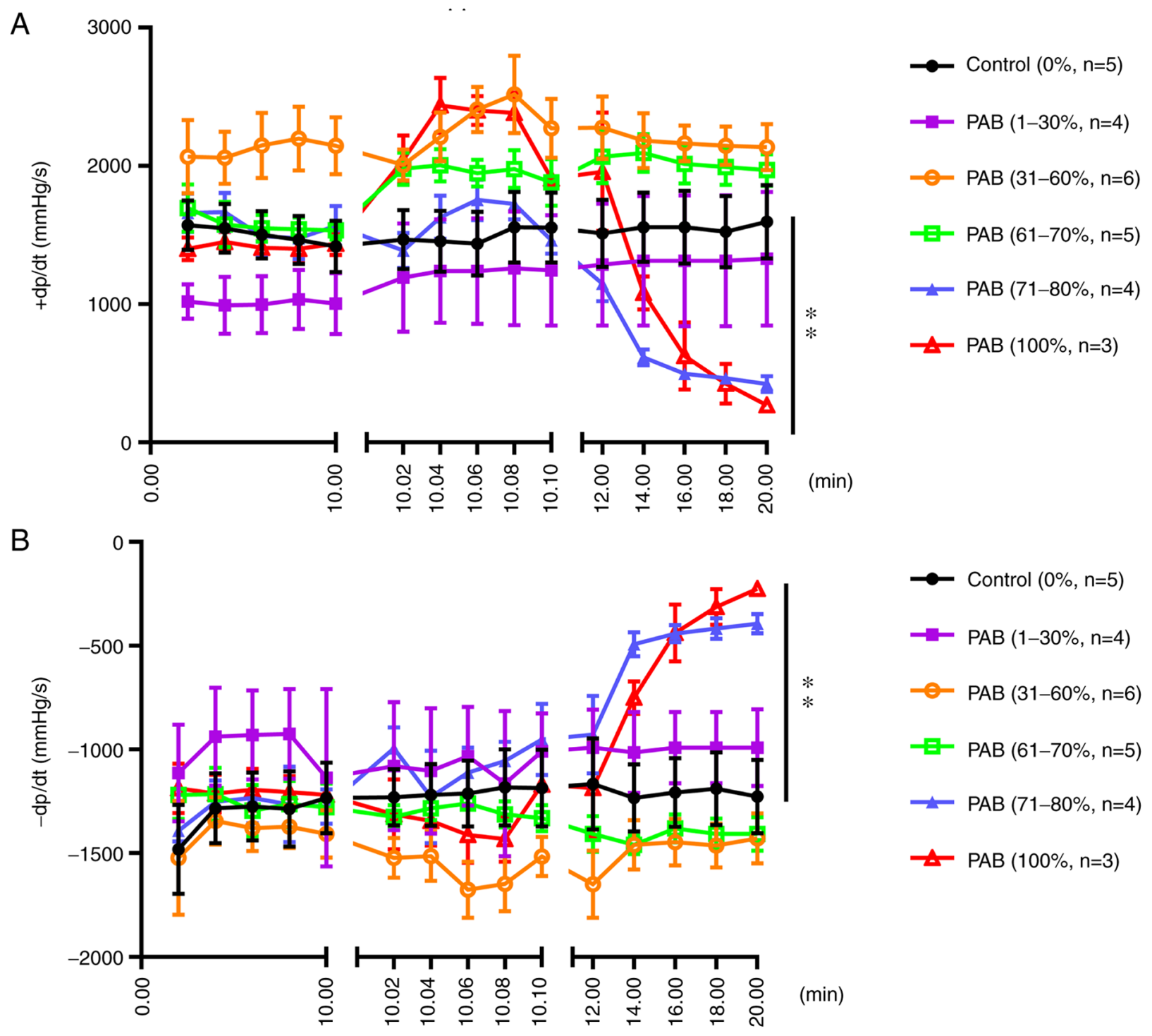

Figure 6. (A) Positive rate of increase in RV pressure (+dp/dt) following gradient PAB. The results of statistical analysis of the differences between groups are as follows in detail: Control vs. PAB (1-30\%), not significant; control vs. PAB (31-60\%), not significant; control vs. PAB (61-70\%), not significant; control vs. PAB (71-80\%), P<0.01; control vs. PAB (100\%), P<0.01. (B) Negative rate of increase in RV pressure (-dp/dt) following gradient PAB. The results of statistical analysis of the differences between groups are as follows in detail: Control vs. PAB (1-30\%), not significant; control vs. PAB (31-60\%), not significant; Control vs. PAB (61-70\%), not significant; control vs. PAB (71-80\%), P<0.01; control vs. PAB (100\%), P<0.01. RV, right ventricle; PAB, pulmonary artery banding. ${ }^{* * *} \mathrm{P}<0.01$.

of hemodynamic and histopathological severity, and is characterized by a high mortality rate (17). However, it differs from human PAH as regards the presentation of an initial permeability lung edema, with the early loss of the endothelial barrier and prominent inflammatory adventitial cell proliferation (18). Furthermore, MCT can cause injury to other organs, such as the liver and kidneys (19); therefore, this model is not the optimal choice for investigating the progression of right heart failure induced by PAH.

In the present study, right heart failure was demonstrated by objectively recording the hemodynamic characteristics of the RV in rats with varying degrees of PAB. There were no significant differences in RV pressure between the PAB (1-30\%), PAB (31-60\%) and the control groups; by contrast, the pressure of the RV increased rapidly and decreased rapidly in 2 sec when the degree of ligation was $71-100 \%$. RVP follows PAH owing to compensatory mechanisms to the increased afterload. Persistent overload results in RV dysfunction and failure and, eventually, death from acute right heart failure ensues. Of note, the RV pressure can be stably maintained at a high level when the degree of ligation is maintained at $61-70 \%$ (Fig. 4). In humans, the pressure of the RV first increases, and then decreases when the RV pressure is overloaded (20). Based on the aforementioned results, it was hypothesized that, in order to design a model of chronic right heart failure, the PAB (1-30\%) and PAB (31-60\%) groups cannot be used, and only the PAB (61-70\%) group would be suitable; in order to construct an acute right heart failure model, the PAB (71-80\%) group appears to be the optimal choice. In the present study, the authors only examined the hemodynamics of the RV following PAB in normal animal models. This may thus constitute a limitation of the present study. In the future, further animal studies are required in order to confirm the effectiveness of this model. 
The authors thus aim to further study the hemodynamics of the RV following PAB in other animal models of pulmonary hypertension in the future.

In conclusion, the present study demonstrates that the hemodynamic characteristics of rats differed with varying degrees of PAB. This finding suggests that the appropriate degree of PAB needs to be selected for different models. As suggested by the present findings, in the chronic model, PAB $61-70 \%$ would be suitable and in the acute model, PAB (71-80\%) group appears to be the optimal choice.

\section{Acknowledgements}

Not applicable.

\section{Funding}

No funding was received.

\section{Availability of data and materials}

The analyzed data sets generated during the study are available from the corresponding author on reasonable request.

\section{Authors' contributions}

NW designed the study. JS, YW, WL and SS contributed to data collection and analysis, and in the manuscript preparation. JS wrote the initial manuscript. NW had primary responsibility for the final content. All authors have read and approved the final manuscript. NW and JS confirm the authenticity of all the raw data.

\section{Ethics approval and consent to participate}

Animal experiments were approved by Gansu Provincial Hospital (approval no. syll20140303).

\section{Patient consent for publication}

Not applicable.

\section{Competing interests}

The authors declare that they have no competing interests.

\section{References}

1. Axente L, Sinescu C and Bazacliu G: Heart failure prognostic model. J Med Life 4: 210-225, 2011.

2. Juenger J, Schellberg D, Kraemer S, Haunstetter A, Zugck C, Herzog W and Haass M: Health related quality of life in patients with congestive heart failure: Comparison with other chronic diseases and relation to functional variables. Heart 87: 235-241, 2002.

3. Clark AL, Johnson M, Fairhurst C, Torgerson D, Cockayne S, Rodgers S, Griffin S, Allgar V, Jones L, Nabb S, et al: Does home oxygen therapy (HOT) in addition to standard care reduce disease severity and improve symptoms in people with chronic heart failure? A randomised trial of home oxygen therapy for patients with chronic heart failure. Health Technol Assess 19: $1-120,2015$.

4. McMurray JJ and Pfeffer MA: Heart failure. Lancet 365: 1877-1889, 2005
5. Dickstein K, Cohen-Solal A, Filippatos G, McMurray JJ, Ponikowski P, Poole-Wilson PA, Stromberg A, van Veldhuisen DJ, Atar D, Hoes AW, et al: ESC guidelines for the diagnosis and treatment of acute and chronic heart failure 2008: The Task Force for the diagnosis and treatment of acute and chronic heart failure 2008 of the European Society of Cardiology. Developed in collaboration with the Heart Failure Association of the ESC (HFA) and endorsed by the European Society of Intensive Care Medicine (ESICM). Eur J Heart Fail 10: 933-989, 2008.

6. Griffiths A, Paracha N, Davies A, Branscombe N, Cowie MR and Sculpher M: The cost effectiveness of ivabradine in the treatment of chronic heart failure from the U.K. National Health Service perspective. Heart 100: 1031-1036, 2014.

7. Lloyd-Jones D, Adams R, Carnethon M, De Simone G, Ferguson TB, Flegal K, Ford E, Furie K, Go A, Greenlund K, et al: Heart disease and stroke statistics-2009 update: A report from the American Heart Association Statistics Committee and Stroke Statistics Subcommittee. Circulation 119: e21-e181, 2009.

8. Vildbrad MD, Andersen A, Holmboe S, Ringgaard S, Nielsen JM and Nielsen-Kudsk JE: Acute effects of levosimendan in experimental models of right ventricular hypertrophy and failure. Pulm Circ 4: 511-519, 2014.

9. Andersen S, Schultz JG, Holmboe S, Axelsen JB, Hansen MS, Lyhne MD, Nielsen-Kudsk JE and Andersen A: A Pulmonary Trunk Banding Model of pressure overload induced right ventricular hypertrophy and failure. J Vis Exp: Nov 29, 2018 (Epub ahead of print). doi: 10.3791/58050.

10. Hirata M, Ousaka D, Arai S, Okuyama M, Tarui S, Kobayashi J, Kasahara S and Sano S: Novel model of Pulmonary Artery Banding leading to right heart failure in rats. Biomed Res Int 2015: 753210, 2015.

11. Bae HK, Lee H, Kim KC and Hong YM: The effect of sildenafil on right ventricular remodeling in a rat model of monocrotaline-induced right ventricular failure. Korean J Pediatr 59: 262-270, 2016.

12. Mendes-Ferreira P, Maia-Rocha C, Adão R, Mendes MJ, Santos-Ribeiro D, Alves BS, Cerqueira RJ, Castro-Chaves P, Lourenço AP, De Keulenaer GW, et al: Neuregulin-1 improves right ventricular function and attenuates experimental pulmonary arterial hypertension. Cardiovasc Res 109: 44-54, 2016.

13. Nogueira-Ferreira R, Vitorino R, Ferreira R and HenriquesCoelho T: Exploring the monocrotaline animal model for the study of pulmonary arterial hypertension: A network approach. Pulm Pharmacol Ther 35: 8-16, 2015.

14. Rain S, Andersen S, Najafi A, Gammelgaard Schultz J, da Silva Goncalves Bos D, Handoko ML, Bogaard HJ, Vonk-Noordegraaf A, Andersen A, van der Velden J, et al: Right ventricular myocardial stiffness in experimental pulmonary arterial hypertension: Relative contribution of fibrosis and myofibril stiffness. Circ Heart Fail 9: e002636, 2016.

15. Campian ME, Hardziyenka M, Michel MC and Tan HL: How valid are animal models to evaluate treatments for pulmonary hypertension? Naunyn Schmiedebergs Arch Pharmacol 373: 391-400, 2006.

16. Gomez-Arroyo JG, Farkas L, Alhussaini AA, Farkas D, Kraskauskas D, Voelkel NF and Bogaard HJ: The monocrotaline model of pulmonary hypertension in perspective. Am J Physiol Lung Cell Mol Physiol 302: L363-L369, 2012.

17. Dickinson MG, Bartelds B, Borgdorff MA and Berger RM: The role of disturbed blood flow in the development of pulmonary arterial hypertension: Lessons from preclinical animal models. Am J Physiol Lung Cell Mol Physiol 305: L1-L14, 2013.

18. Naeije R and Dewachter L: Animal models of pulmonary arterial hypertension. Rev Mal Respir 24: 481-496, 2007 (In French).

19. Yao J, Li CG, Gong LK, Feng CC, Li CZ, Gao M, Luan Y, Qi XM and Ren J: Hepatic cytochrome P450s play a major role in monocrotaline-induced renal toxicity in mice. Acta Pharmacol Sin 35: 292-300, 2014

20. Harjola VP, Mebazaa A, Čelutkienè J, Bettex D, Bueno H, Chioncel O, Crespo-Leiro MG, Falk V, Filippatos G, Gibbs S, et al: Contemporary management of acute right ventricular failure: A statement from the Heart Failure Association and the Working Group on Pulmonary Circulation and Right Ventricular Function of the European Society of Cardiology. Eur J Heart Fail 18: 226-241, 2016.

This work is licensed under a Creative Commons Attribution-NonCommercial-NoDerivatives 4.0 International (CC BY-NC-ND 4.0) License. 